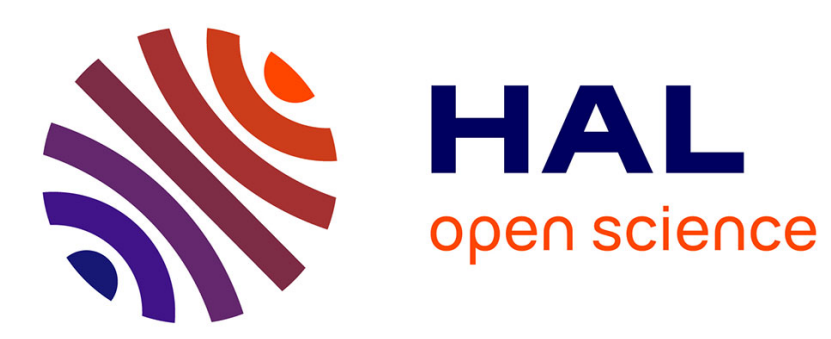

\title{
Realistic simulation of surface defects in 5-axis milling using the measured geometry of the tool
}

\author{
Sylvain Lavernhe, Yann Quinsat, Claire Lartigue, Christopher Brown
}

\section{To cite this version:}

Sylvain Lavernhe, Yann Quinsat, Claire Lartigue, Christopher Brown. Realistic simulation of surface defects in 5-axis milling using the measured geometry of the tool. International Journal of Advanced Manufacturing Technology, 2014, 74, pp. 393-401. 10.1007/s00170-014-5689-7 . hal-01002334

\section{HAL Id: hal-01002334 \\ https://hal.science/hal-01002334}

Submitted on 5 Jun 2014

HAL is a multi-disciplinary open access archive for the deposit and dissemination of scientific research documents, whether they are published or not. The documents may come from teaching and research institutions in France or abroad, or from public or private research centers.
L'archive ouverte pluridisciplinaire HAL, est destinée au dépôt et à la diffusion de documents scientifiques de niveau recherche, publiés ou non, émanant des établissements d'enseignement et de recherche français ou étrangers, des laboratoires publics ou privés. 


\title{
Realistic simulation of surface defects in 5-axis milling using the measured geometry of the tool
}

\author{
Sylvain Lavernhe · Yann Quinsat · Claire Lartigue · Christopher Brown
}

Received: date / Accepted: date

\begin{abstract}
Managing macro and micro geometry of surfaces during manufacturing processes is a key factor for their following uses. Indeed micro-geometry and surface topography are directly linked to the performances of functions (contact, friction, lubrication, etc.) by texture parameters to ensure the desired local geometry. Common models for simulation of surface topography are based on ideal geometry of the machining tool and cannot represent surface defects. The actual prediction and simulation of defects is one step forward in a competitive context. In this paper, the realistic model proposed aims to simulate and predict as finely as possible local defects of machined surfaces taking into account the actual edge geometry of the cutting tool. The combined use of the machining kinematics and of the measured geometry of the cutting edges leads to the representation of the geometrical envelope of the surface using a Zbuffer technique. Simulation assessment is carried out by the analysis of 3D surface topography parameters such as surface complexity and relative area, and by a comparison of simulation results to an experimental case of study.
\end{abstract}

Keywords Surface topography · Defects · Realistic modeling and simulation - Surface analysis - Surface machining

S. Lavernhe - Y. Quinsat - C. Lartigue

LURPA

ENS de Cachan - Université Paris Sud 11

61 avenue du Président Wilson, 94235 Cachan cedex, France

Tel.: +33147402985

Fax: +331474022 20

E-mail: lavernhe@lurpa.ens-cachan.fr

C. Brown

Surface Metrology Laboratory

Mechanical Engineering Department, Worcester Polytechnic Institute

Worcester, MA, 01609 USA

E-mail: brown@wpi.edu

\section{Introduction}

Surface topography characterization resulting from 5 -axis milling is a current and essential issue. Some studies have enhanced the importance of 3D surface topography in engineering applications. 3D surface topography influences mechanical and physical properties of contacting parts and plays a major role in surface integrity, which affects fatigue life $[21,18]$. The defect size distribution, as well as the defect orientation, are of great interest for a better understanding of the mechanical behavior under loads.

The characterization of surface topography as a function of cutting conditions is thus a challenge, in particular for the prediction of the shape, size and orientation of the defects induced by machining. Several authors handle this issue through simulations. Most simulations concern 3-axis ball-end milling and, are based on the 3D modeling of both the tool geometry and the workpiece. Kim and Chu [14] described the texture obtained in milling by using a texture superposition model considering the effect of the fillet radius. In this study, the authors investigate the influence of the feedrate, the cutter type, and the run-out. Bouzakis et al. focused on the motion of the cutting edge, which is segmented into elementary linear sections of constant width. These authors highlight the influence of the tool orientation, the transverse step and the feedrate on the machined surface quality [8]. Liu et al. detailed a method based on the solid modeling of each cutter flute [17]. The workpiece surface is sampled in the (XY) plane by a set of parallel lines (called spikes) to the $\mathrm{Z}$ axis (tool axis). The cutter tool generates a volume which truncates the spikes. The remaining parts of the reference spikes provide a good approximation of the surface finish which can be linked with the cutting parameters. In a previous work, we proposed to link the machining strategy in 3-axis ball-end milling with a 3D surface roughness parameter and to optimize the machining direction ac- 
cording to this parameter [19]. In their work, Arizmendi et al. [5] proposed a model accounting for the tool parallel axis offset. The cutting edge geometry is represented as a cylindrical helix projected onto a spherical surface. The topography prediction is thus obtained considering the equation of the cutting edge trajectory and the equation of the material swept by the tool. More recently, Buj-Corral et al. [9] focused on the prediction of roughness generated in ball-end milling of flat surfaces. The surface is modeled as a set of horizontal planes (the vertical $\mathrm{Z}$ defining the tool axis), each of them sampled according to a square grid (X, Y). Each cutting tool tooth is modeled as an elliptical curve of increasing radius. The topography is built based on a set of intersections between the tool motion and the part. The effect of cutting conditions is taken into account as well as the effect of some characteristic of the tool geometry. In their approach, Denkena et al. [10] considered the surface topography as the sum of the "kinematic topography", resulting from the cutting edge movement, and the "stochastic topography", including surface irregularities and cracks. The first one is obtained using Boolean operations, simulating material removal, between the CAD-model of the tool and a discrete model of the workpiece. The "stochastic topography" is generally obtained by subtracting the kinematic topography to surface measurements. Nevertheless, the authors proposed a method to evaluate this stochastic topography with an empirical model based on the process force simulations.

Few works handle the case of 5-axis milling. Kim et al. [14] proposed to simulate the 3D topography obtained in 5axis milling by using a filleted-ball end tool. The envelope of the tool movement is modeled by successive tool locations according to the feed per tooth. Antoniadis et al. [4] consider the general case of multi-axis with ball-end tools. The cutting edge of the tool is decomposed into an elementary cutting edge of trapezoidal shape. The workpiece is modeled as a set of linear segments (or needles) defining a brush. The geometrical model of the cutting tool follows the selected milling operation and intersects the set of needles. The part material remaining defines the milled surface and gives its 3D topography. Starting from the expression of the trajectory equation of the cutting edge relative to the workpiece and the tool paths, Zhang et al. introduced a new and general iterative method to simulate roughness in multi-axis ballend milling $[11,23]$. The authors investigated in particular the effects of the tool inclination and of the cutting mode on surface roughness. Indeed, their study showed that surface roughness remains mostly unchanged beyond a certain limiting inclination angle, and that the cutting mode influences mainly sculptured surface roughness.

In the case of 5-axis milling, we proposed in a first study a theoretical approach to simulate the 3D surface topography obtained in 5-axis milling with a filleted-ball end cutter tool integrating actual feedrate evolution. The material removal simulation relies on the well-known N-buffer method and requires the modeling of the tool geometry, the surface and the actual tool trajectory $[16,20]$. The surface is discretized according to a rectangular grid with lines directed along the local normal to the surface. The set of lines is truncated by the envelope of the tool trajectory leading to the $3 \mathrm{D}$ surface topography (Fig. 1).

The local actual feedrate evolution is obtained by a kinematical predictive model. The tool is assumed to be rigid. It is approximated by a local meshing built from the CAD theoretical model of the tool geometry. The comparison between simulations and experimental trials shows good agreement between the predicted 3D topography and the experimental one (Fig. 2).

Nevertheless, and as for most methods available in literature, the modeling of the cutting process only attempts geometrical fidelity on scales of the curvature of a tool with a smooth cutting edge. This leads to a good representation of the general pattern, but the model is not able to predict small defects appearing at the bottom of the valleys (Fig. 3).

However, the knowledge of the size and distribution of the defects is essential in fracture mechanics to establish the relationship between defect distribution and the fracture probability. Studies have enhanced the effect of microgeometry on fatigue life [18] [12]. Fatigue failure of a mechanical part can originate from micro-scratches on the surface. By considering the statistical defect size distribution that exists in an effective volume, it is possible to calculate the failure probability of the structure. For small defects (less than $5 \mu \mathrm{m}$ ) Guillemot and Souto-Lebel et al. [21] proposed to model the defect size population as a beta function and used this model to identify the defect distribution leading to experimental fatigue limits and compared it favorably to the distribution obtained by direct measurement.

In addition, the general direction of the texture lay or anisotropy is of great importance with regard to the load direction. To predict actual surface topography, in contrast with Dekena et al., one can consider that the difference between the measured topography and the topography that was simulated using classical tools (also called "kinematic topography") is not stochastic. The difference might rather be due to finer scale phenomena not taken into account in simulation, e.g., actual tool geometry, and tool deflection. In fact, as displayed in Fig. 3, local defects in the bottom of the valleys seem to repeat in the machining (or feed) direction. It can be observed a large population of defects smaller than $10 \mu \mathrm{m}$. This is probably related to defects of the edge of the cutting tool. Such defects are not predictable if the geometry of the cutting edge is supposed to be smooth. A change of scale is required.

Therefore, the prediction of local defects requires the modeling of the tool surface characteristics at a finer scale, more similar to that used for grinding $[13,6]$. Generally 


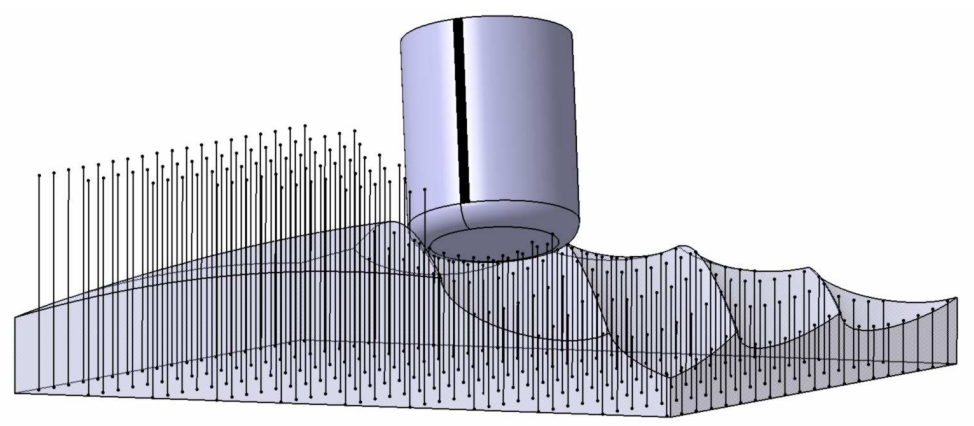

Fig. $13 D$ surface topography simulation in 5 axis-milling



Fig. 2 Surface topography: simulated (left) and rendering of a measurement of an actual machined surface (right)



Fig. 3 Actual defects in the bottom of the valleys

speaking, most simulations of the grinding process are based on models describing the macro- and micro-geometry of the grinding wheel, the grit size, the grit volume, and the grit distribution. These parameters are obtained from an experimental characterization of the grinding tool. Actually, the cutting edge distribution used to simulate the relative motion between the grinding wheel and the workpiece is obtained by measuring the wheel topography. As a result, surface roughness predictions are in good agreement with experimental ones.

In a similar way, we propose to improve 3D surface topography prediction in milling by characterizing the actual cutting edge of the tool. For this purpose, the tool cutting edge is measured using an Alicona infinite focus system which measures the actual topography of the cutting edge. The latter is integrated in the simulation method previously implemented for 5-axis milling surface topography prediction to account for the measured topography of the tool. 


\section{Model for surface topography simulation with measured tool topography}

The material removal simulation relies on the well-known $\mathrm{N}$-buffer method and requires the modeling of the surface, the tool geometry and the actual tool trajectory $[16,20]$.

The surface is discretized according to a rectangular grid. At each point of the grid, a line directed along the local normal to the surface is defined. The set of lines is truncated by the envelope of the tool trajectory, and the remaining part defines the 3D topography of the machined surface (Fig. 1).

The tool, considered as rigid, is approximated as a local mesh, expressed in the STL format. Only active cutting edges are considered to reduce the number of nodes and so the calculation time. In previous works, these cutting edges were assumed to be smooth. More details on the mesh characteristics are given in section 3. For each simulation step of the model, the mesh is transformed versus the workpiece according the kinematics of the 5-axis trajectory.

Concerning the tool trajectory, the input of the material simulation model is the actual tool trajectory expressed as a set of tool postures and corresponding feedrates. The latter are calculated based on a model of velocity prediction which gives a good estimation of the local feedrate of the tool teeth [15].

The simulation of material removal thus consists of two main steps:

1. calculation of the spindle angular positions at each tool posture

2. trajectory sampling preceding the N-buffer computation

Based on a linear interpolation, of the tool trajectory between the local postures $\left\{X_{p}, Y_{p}, Z_{p}\right\}$, the time interval $\Delta t$ separating two postures is calculated as follows:

$$
\Delta L^{i, i+1}=\sqrt{\left(X_{p}^{i+1}-X_{p}^{i}\right)^{2}+\left(Y_{p}^{i+1}-Y_{p}^{i}\right)^{2}+\left(Z_{p}^{i+1}-Z_{p}^{i}\right)^{2}}
$$

$\Delta t^{i, i+1}=\frac{\Delta L^{i, i+1}}{\frac{V_{f}^{i+1}-V_{f}^{i}}{2}}$

in which the local feedrate $V_{f}$ is linearly interpolated.

Let $\Omega_{\text {spindle }}$ be the rotational velocity of the spindle, supposed to be constant regardless of fluctuations in the actual feedrate, angular positions of the tool axis $\alpha$ are given by:

$\alpha^{i+1}=\alpha^{i}+\Omega_{\text {spindle }} \cdot \Delta t^{i, i+1}$

The elementary trajectory defined between two tool postures is checked considering a fixed geometrical step $d \alpha$ to ensure a good discretized description of the envelop of the tool movement:

$d t=\frac{d \alpha}{\Omega_{\text {spindle }}}$
Given this elementary time step $d t$, it is thus possible to discretize the time interval $\Delta t$ and determine the number of samples $N_{\text {samples }}$ for each linear interpolation by the integer part and then the current sampling number $N^{*}$ :

$N^{*} \in\left[\left[0 \ldots N_{\text {samples }}^{i, i+1}\right] \quad\right.$ with $\quad N_{\text {samples }}^{i, i+1}=\left\lfloor\frac{\Delta t^{i, i+1}}{d t}\right\rfloor$

This is used in the calculation of the sampled tool velocity $V_{f}^{*}$ and locations of the components $X_{p}^{*}, Y_{p}^{*}$ or $Z_{p}^{*}$ along the elementary trajectory:

$V_{f}^{*}=V_{f}^{i}+\frac{V_{f}^{i+1}-V_{f}^{i}}{\Delta t^{i, i+1}} \cdot N^{*} \cdot d t$

$X_{p}^{*}=X_{p}^{i}+d X^{*}=X_{p}^{i}+\frac{X_{p}^{i+1}-X_{p}^{i}}{\Delta L^{i, i+1}} \cdot \frac{V_{f}^{*}+V_{f}^{i}}{2} \cdot N^{*} \cdot d t$

The components $I^{*}, J^{*}$ and $K^{*}$ of the tool axis orientation $\mathbf{u}$ are computed so that the orientation vector of the tool runs in a plane created by the start and end vectors $\mathbf{u}^{\mathbf{i}}$ and $\mathbf{u}^{\mathbf{i}+\mathbf{1}}$ for each linear interpolation. The rotation angle $\theta^{*}$ between the start vector $\mathbf{u}^{\mathbf{i}}$ and an intermediate orientation $\mathbf{u}^{*}$ respects the ratio between the angle and the linear displacement covered:

$\theta^{*}=\theta^{i, i+1} \cdot N^{*} \cdot \frac{d t}{\Delta t^{i, i+1}}$

The intermediate orientation vector $\mathbf{u}^{*}$ is given by the Rodrigues' rotation formula, Eq. 9, with $\mathbf{w}$ the unit vector defining the axis of rotation (cross product of the start and end vectors).

$\mathbf{u}^{*}=\mathbf{u}^{\mathbf{i}} \cos \theta^{*}+\left(\mathbf{w} \times \mathbf{u}^{\mathbf{i}}\right) \sin \theta^{*}+\mathbf{w}\left(\mathbf{w} \cdot \mathbf{u}^{\mathbf{i}}\right)\left(1-\cos \theta^{*}\right)$

The interpolated angular position of the tool $\alpha^{*}$ is directly given by:

$\alpha^{*}=\alpha^{i}+\Omega_{\text {spindle }} \cdot N^{*} \cdot d t$

Finally, the simulated machined surface is obtained by computing the intersections between the normal lines representing the surface and the tool for each configuration $\left\{X_{p}^{*}, Y_{p}^{*}, Z_{p}^{*}, I^{*}, J^{*}, K^{*}, \alpha^{*}\right\}$.

The whole simulation method for material removal is summarized in Fig. 4. This model leads to simulations in good agreement with experimental results, in 3-axis as well as 5-axis milling and regardless of the tool geometry [16]. However, as aforementioned, the model failed in the prediction of chaotic small defects. The main reason is likely due to the ideal modeling of the tool cutting edges. Therefore, the simulation is improved by integrating a realistic description of the cutting edge instead of the perfect geometrical description. 


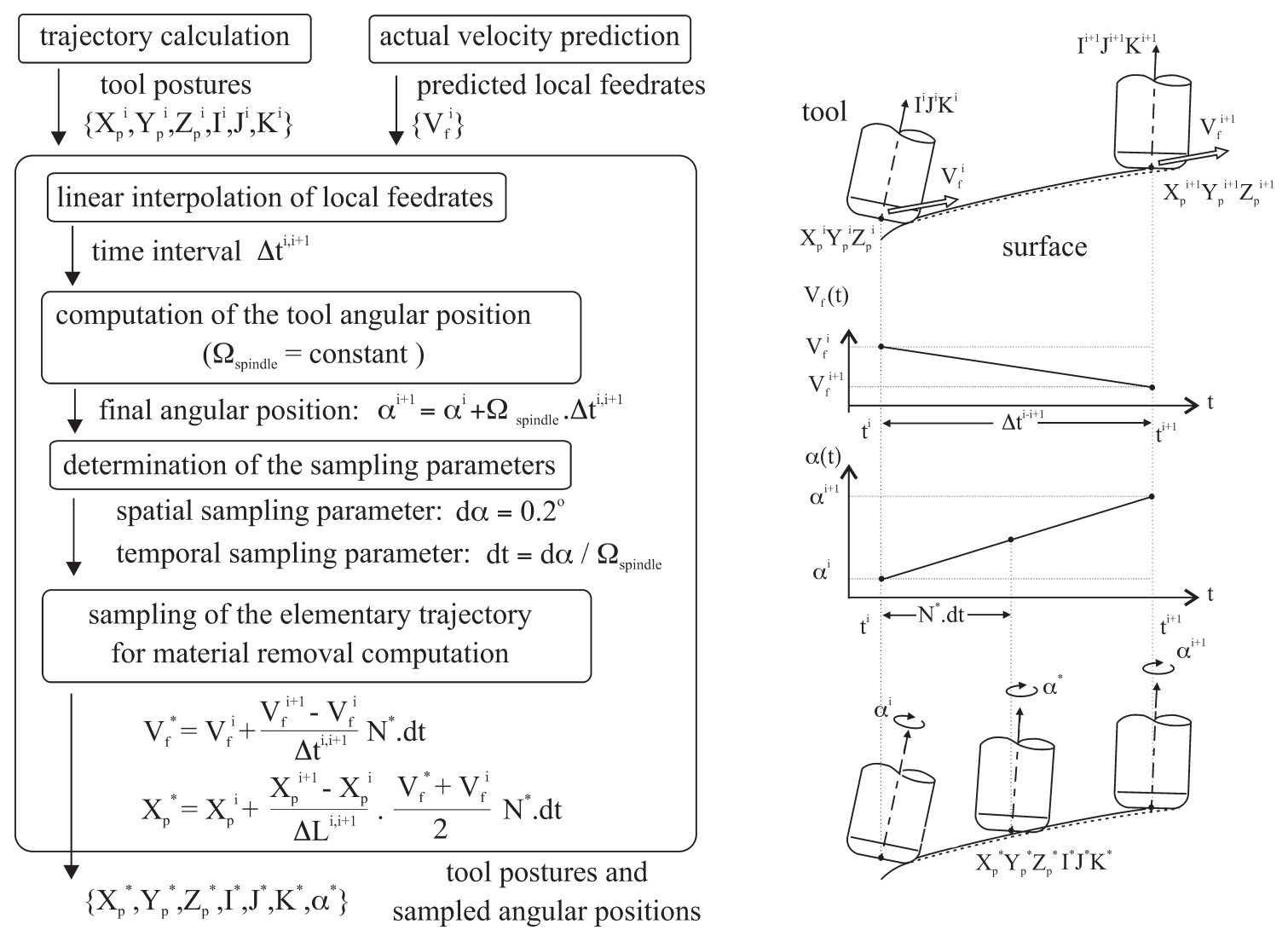

Fig. 4 Material removal simulation process

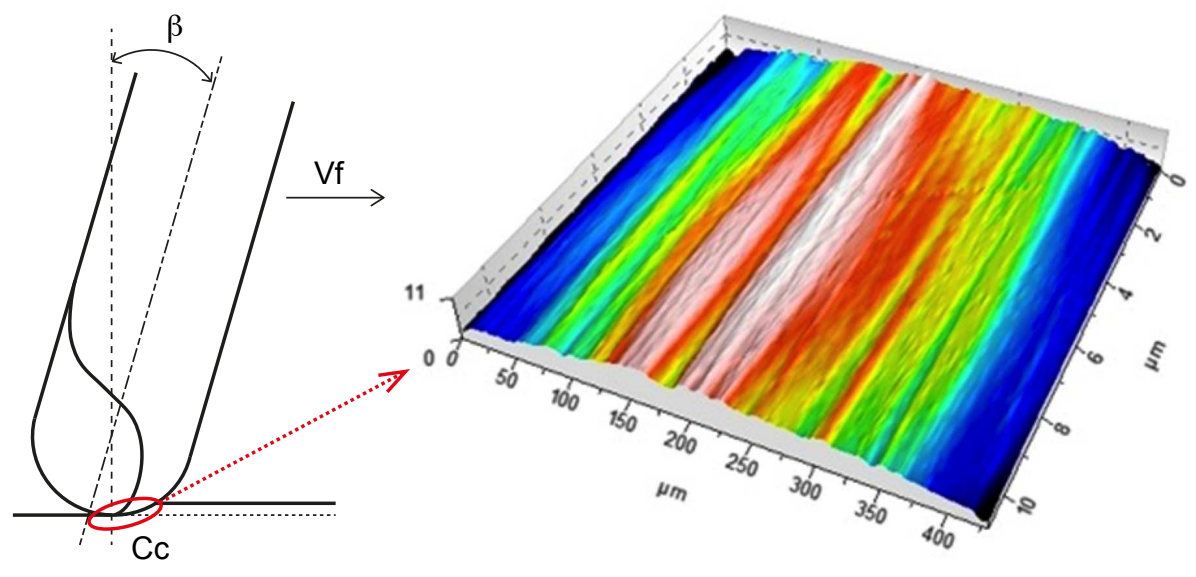

Fig. 5 Description of the measured cutting edge

\section{Experimental set-up}

The actual geometry of the cutting edge is only accessible through measurement. For this purpose, once the tool is selected its cutting edges have to be measured before machining. To control the process better, it is easier to consider just one cutting edge. Therefore, only the track associated to this tooth will be left on the part surface after machining. To illustrate our purpose, the machining of a plane surface with a ball ended tool inclined by an tilt angle $\beta$ in the feed direction $V_{f}$ is proposed (Fig. 5).

The tool cutting edge is measured using an Alicona infinite focus. Data provided by the manufacturer state the best vertical resolution of the measurement system is $20 \mathrm{~nm}$ and the vertical repeatability is $8 \mathrm{~nm}$. For the simulation, and to limit the number of points (18790 points), only the functional part around the contact point $C_{c}$ of the cutting edge is considered. This functional portion of the cutting edge is 


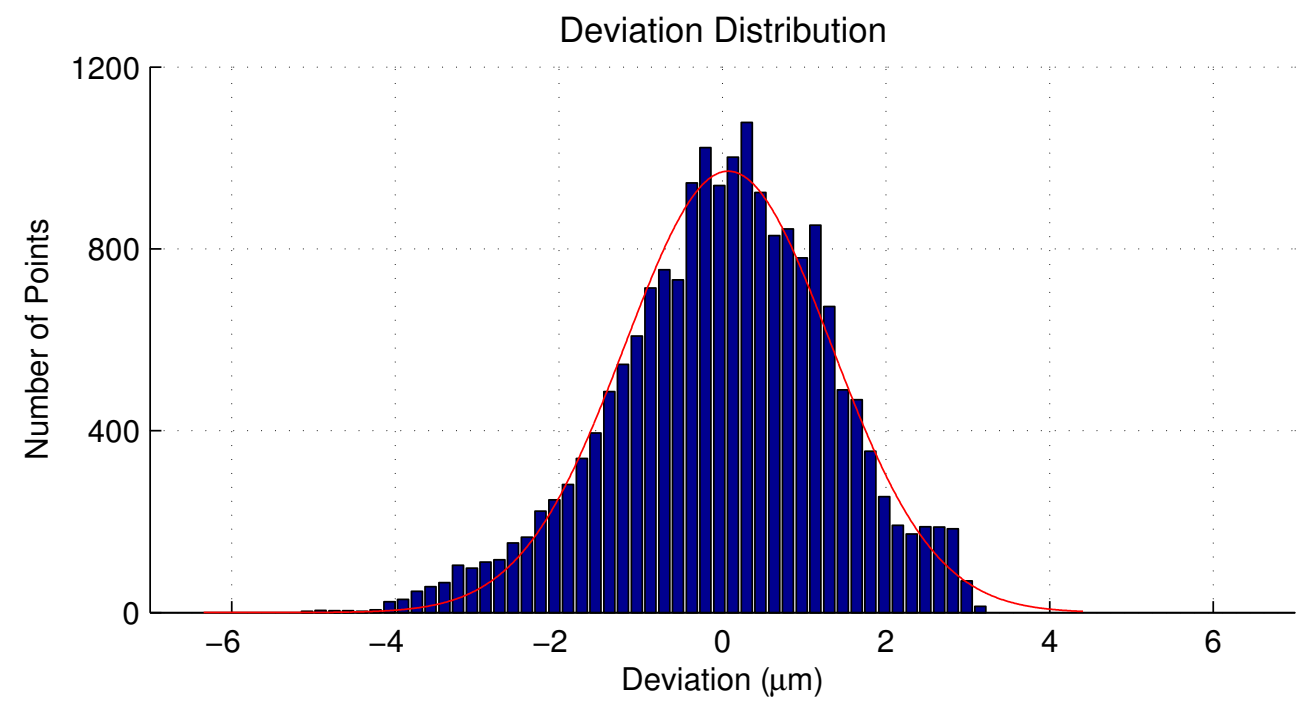

Fig. 6 Mesh point distribution

Table 1 Mesh description

\begin{tabular}{lll}
\hline & Edge length $(\mu m)$ & Face area $\left(\mu m^{2}\right)$ \\
\hline Mean & 0.79 & 0.25 \\
\hline Standart Deviation & 0.23 & 0.10 \\
\hline
\end{tabular}

$\delta=R \cdot\left(1-\cos \left(\frac{\alpha}{2}\right)\right)$

meshed in a STL format giving 36389 facets. The dimensional characteristics of the mesh used are given in table 1. The analysis of noise on the mesh is not easy. Indeed, the study of the distribution of points around a theoretical sphere (Fig. 3) shows that it is difficult to differentiate the points associated to the cutting edge from those corresponding to the rake face and flank face. However, given the characteristics of the measuring system used, the resulting mesh is compatible with our need.

A specimen of high-strength steel (50CrMo4 bainitic steel see Tab. 2 for specimen mechanical properties) is machined in 5 axes with a constant tilt angle is taken egal to $20^{\circ}$ on a HSM milling center using a ball-ended tool $(R=5 \mathrm{~mm})$. The cutting conditions $\left(\Omega_{\text {spindle }}=18000 \mathrm{rpm}\right.$, feed per tooth $0.2 \mathrm{~mm}$ ) are chosen according to the tool manufacturer recommendations. The machined specimen is then measured with a chromatic confocal Micromeasure station.

Simulation parameters are chosen in order to generate deviations with a magnitude lower than the measurement noise, and that is therefore studied. Hence, considering the tool radius of $5 \mathrm{~mm}$, the angular step $d \alpha=0.2^{\circ}$ generates a chordal deviation $\delta$ under $10 \mathrm{~nm}$ (Eq.11). As the linear interpolation of the trajectory is based on this parameter $(d \alpha)$, its does not add additional geometrical error on the surface topography.

\section{Results and discussion}

Results of the measurement and of the simulation are displayed in Fig. 7. To highlight the contributions of the proposed method, a surface compound of spherical caps is added. This surface is computed according to the models proposed by Kim et al. [14] and Quinsat et al. [19]. As it can be seen, the simulation using the measure tool profile appears to be a good estimation of the actual surface pattern (measured), although the amplitude of the peaks is slightly over-estimated. Actually, if only the track of the sphere is considered (Fig. 7right), the simulation lacks any of fine scale details evident on the measured surface.

The apparent improvement in the simulation achieved by using the measured tool profile is confirmed by considering the roughness parameters: relative area area-scale complexity. These standardized parameters [2] relate to the inclinations on the surface and account for the complexity of the surface shapes at different scales. In fact, the idea is to exploit the chaotic, or fractal, nature of the surface, in particular that the apparent area of a surface depends on the scale of observation. In the area-scale analysis the topography of the surface is tiled using triangular patches. At any particular considered scale, all the patches have the same area (defining the scale), and the relative area at the scale is the calculated area divided by the nominal area [7, 22, 1]. The calculated area is given by the number of patches at the considered scale multiplied by the area of the patch at 
Table 2 Mechanical properties of the bainitic steel used for the trial

\begin{tabular}{lllllll}
\hline$E(M P a)$ & $R_{m}(M P a)$ & $\Sigma_{v}(M P a)$ & $C(M P a)$ & $g$ & $A(\%)$ & $\operatorname{Hardness}(H V)$ \\
\hline 190000 & 1080 & 480 & 188000 & 580 & 17 & 315 \\
\hline
\end{tabular}


Fig. 7 3D obtained pattern (left-measured; middle-simulation; right-sphere track)

the scale. The area-scale complexity is the slope of the relative area plot. It can be calculated between any two scales where the tiling is performed. If the surfaces can be considered multi-fractal with respect to scale, then this complexity is related to the fractal dimension [1]. These parameters, which were calculated using scale-sensitive fractal analysis [3], are displayed in figures 8 and 9. The relative area at different scales clearly highlights that the model based on the smooth sphere is not a good representation of the actual machined surface. At large scales, the simulation with the measured tool profile provides a better representation of the actual surface. On the other hand, at fine scales, the simulation leads to higher values of the relative area, which could be associated with the difference in the peak-to-valley magnitude previously observed. Actually, some effects, such as, abrasion and elastic strains of the material below the cutting edge present during the cutting process, are not taken into account in the simulation.

The complexity of the simulation based on the tool profile (realistic simulation) reaches a maximum at $24.68 \mu^{2}$ and then declines sharply at finer scales This could be because of the refinement of the mesh to a liner scale of about $7 \mu \mathrm{m}$, which corresponds to the leg of a triangle with an area of about $24.5 \mu \mathrm{m}^{2}$. The complexity of the measured surface drops of sharply at scales below $1 \mu \mathrm{m}^{2}$, corresponding to linear scales of about the square root of two. As the value of the diameter spot is equal to $2 \mu \mathrm{m}$, the complexity falls at small scales. Once more, the topography model based on the smooth spherical tool (spherical caps) has a complexity that is as much as two orders of magnitude below the measured topography.
This remark is confirmed by conventional roughness parameter evaluation (Tab. 3). To only consider conventional roughness characterization parameters, the surfaces are filtered using a Gaussian filter $\left(\lambda_{\text {cutoff }}=0.08 \mathrm{~mm}\right)$. Results clearly highlight a better agreement of the value of the conventional characterization parameters obtained using the realistic simulation with those obtained by measurement. The simulation with only spherical caps is not sufficient to account for fine scale surface topography. Therefore, the simulation gives a good estimation of the surface roughness that could be used to link surface integrity with the fatigue-life of the part.

\section{Conclusion}

In the paper, an algorithm for a more realistic simulation of surface formation is proposed. This simulation relies on the well-known Z-buffer method for which a tool cuts a set of vertical spikes representing the part surface. To account for the real tool geometry, the tool cutting edge is measured using an optical means that gives a good representation of the actual edge. To assess the method, a steel workpiece is machined by 3-axis ball-end milling. Comparisons between the 3D topography obtained by realistic simulation to the measured one highlights that the simulation leads to a better estimation of the topography than does a simulation with a smooth tool. In particular, the multi-scale study of the relative area clearly shows that the simulation provides a better representation of the topography than a smooth, sphere-cap tool model. This gives a good estimation of the motif left on the part surface for a scale that can be useful to study 


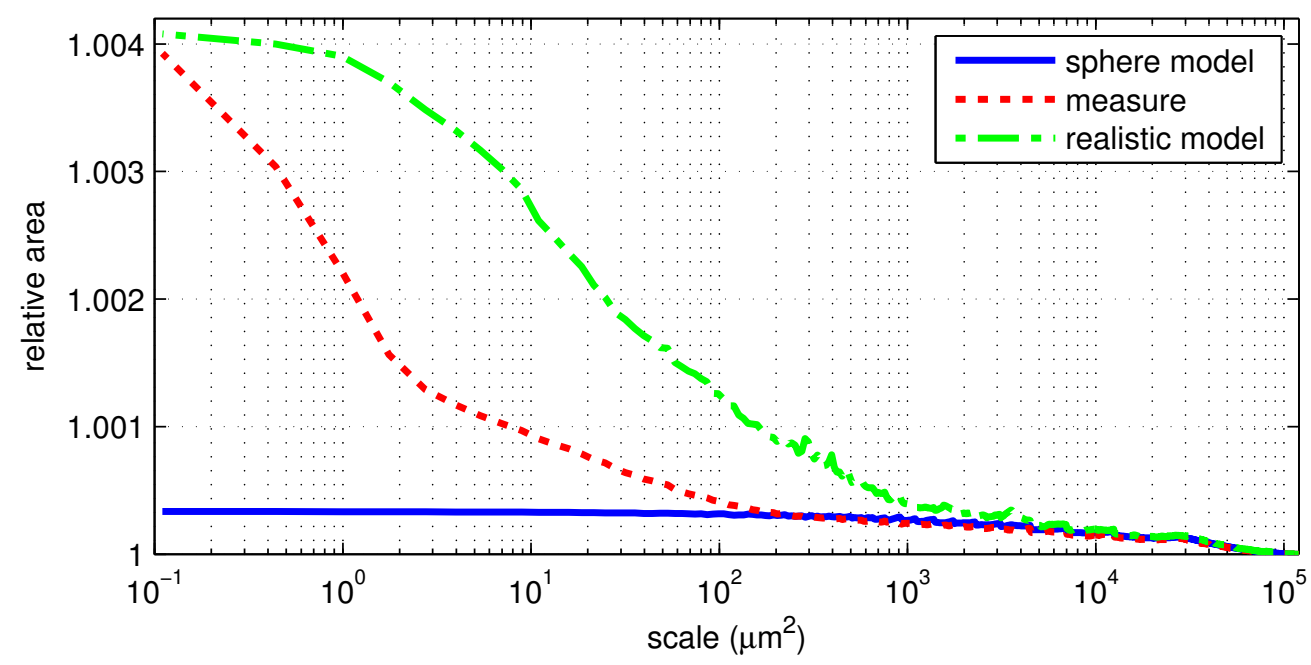

Fig. 8 Relative area vs scale

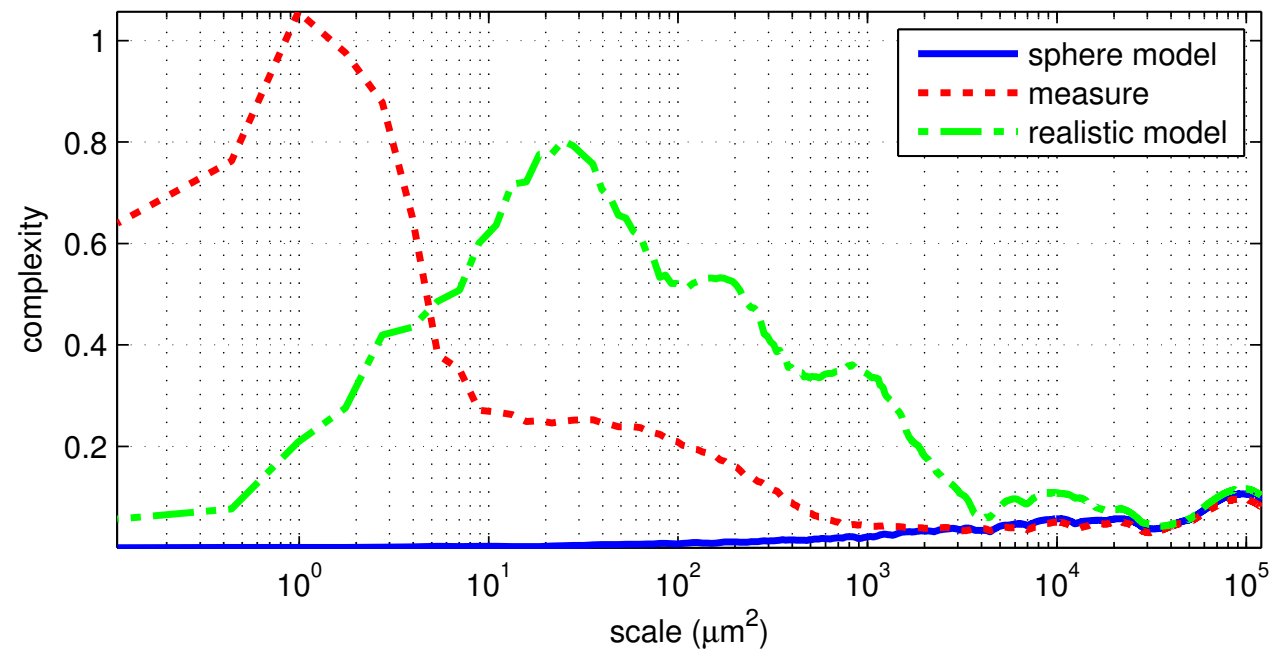

Fig. 9 Complexity vs Scale

Table 3 Amplitude roughness parameters [2]

\begin{tabular}{llllllll}
\hline & $S q(\mu m)$ & $S s k$ & $S k u$ & $S p(\mu m)$ & $S v(\mu m)$ & $S z(\mu m)$ & $S a(\mu m)$ \\
\hline Measured & 0.158 & -0.0281 & 2.85 & 0.515 & 0.825 & 1.34 & 0.127 \\
\hline Realistic simulation & 0.326 & 0.488 & 2.90 & 0.855 & 0.611 & 1.47 & 0.258 \\
\hline Spherical caps & 0.0257 & 2.34 & 7.64 & 0.105 & 0.0153 & 0.120 & 0.0177 \\
\hline
\end{tabular}

the link between surface integrity and fatigue-life. However, the surface complexity obtained by simulation is lower than that obtained for the measured one likely due to calculation limits. A sensitive study of the effect of the tool meshing combined with the effect of the part meshing would give the adequate compromise calculation time/ quality of the representation. It would be interesting to complete the modeling by considering the mechanical behavior of the part under cutting forces. Accounting for the local part elasticity during tool cutting would likely lead to a better estimation of the peak magnitude. 


\section{References}

1. (2009) ASME B46.1: Surface Texture (Surface Roughness, Waviness, and Lay). Revision of ASME B46.12002

2. (2012) ISO 25178-2: Geometrical product specifications (GPS) - Surface texture: Areal - Part 2: Terms, definitions and surface texture parameters. ICS : 17.040.20

3. (2013) SFrax: Surface Metrology and Fractal Analysis - http://www.surfract.com/. URL http://www.surfract.com/

4. Antoniadis A, Savakis C, Bilalis N, Balouktsis A (2003) Prediction of surface topomorphy and roughness in ball-end milling. The International Journal of Advanced Manufacturing Technology 21(12):965-971, DOI 10.1007/s00170-002-1418-8

5. Arizmendi M, Fernández J, de Lacalle LL, Lamikiz A, Gil A, Sánchez J, Campa F, Veiga F (2008) Model development for the prediction of surface topography generated by ball-end mills taking into account the tool parallel axis offset. experimental validation. CIRP Annals - Manufacturing Technology 57(1):101 - 104, DOI 10.1016/j.cirp.2008.03.045

6. Aurich J, Braun O, Warnecke G, Cronjäger L (2003) Development of a superabrasive grinding wheel with defined grain structure using kinematic simulation. CIRP Annals - Manufacturing Technology 52(1):275 280, DOI 10.1016/S0007-8506(07)60583-6

7. Berglund J, Brown C, Rosén BG, Bay N (2010) Milled die steel surface roughness correlation with steel sheet friction. CIRP Annals - Manufacturing Technology 59(1):577 - 580, DOI 10.1016/j.cirp.2010.03.140

8. Bouzakis KD, Aichouh P, Efstathiou K (2003) Determination of the chip geometry, cutting force and roughness in free form surfaces finishing milling, with ball end tools. International Journal of Machine Tools and Manufacture 43(5):499 - 514, DOI 10.1016/S08906955(02)00265-1

9. Buj-Corral I, Vivancos-Calvet J, Domínguez-Fernández A (2012) Surface topography in ball-end milling processes as a function of feed per tooth and radial depth of cut. International Journal of Machine Tools and Manufacture 53(1):151 - 159, DOI 10.1016/j.ijmachtools.2011.10.006

10. Denkena B, Böß V, Nespor D, Samp A (2011) Kinematic and stochastic surface topography of machined TiAl6V4-parts by means of ball nose end milling. Procedia Engineering 19(0):81 - 87, DOI 10.1016/j.proeng.2011.11.083, 1st CIRP Conference on Surface Integrity (CSI)

11. Gao T, Zhang W, Qiu K, Wan M (2005) A new algorithm for the numerical simulation of machined surface topography in multiaxis ball-end milling. Journal of Manufacturing Science and Engineering 128(1):96103, DOI 10.1115/1.2123047

12. Guillemot N, Lartigue C, Billardon R, Mawussi B (2010) Prediction of the endurance limit taking account of the microgeometry after finishing milling. International Journal on Interactive Design and Manufacturing 4(4):239-249, DOI 10.1007/s12008-010-0104-6

13. Inasaki I (1996) Grinding process simulation based on the wheel topography measurement. CIRP Annals - Manufacturing Technology 45(1):347 - 350, DOI 10.1016/S0007-8506(07)63077-7

14. Kim B, Chu C (1999) Texture prediction of milled surfaces using texture superposition method. ComputerAided Design 31(8):485 - 494, DOI 10.1016/S00104485(99)00045-7

15. Lavernhe S, Tournier C, Lartigue C (2008) Kinematical performance prediction in multi-axis machining for process planning optimization. The International Journal of Advanced Manufacturing Technology 37(5-6):534544, DOI 10.1007/s00170-007-1001-4

16. Lavernhe S, Quinsat Y, Lartigue C (2010) Model for the prediction of 3D surface topography in 5-axis milling. The International Journal of Advanced Manufacturing Technology 51(9-12):915-924, DOI 10.1007/s00170010-2686-3

17. Liu N, Loftus M, Whitten A (2005) Surface finish visualisation in high speed, ball nose milling applications. International Journal of Machine Tools and Manufacture 45(10):1152 - 1161, DOI 10.1016/j.ijmachtools.2004.12.007

18. Novovic D, Dewes R, Aspinwall D, Voice W, Bowen P (2004) The effect of machined topography and integrity on fatigue life. International Journal of Machine Tools and Manufacture 44(2 - 3):125 - 134, DOI 10.1016/j.ijmachtools.2003.10.018

19. Quinsat Y, Sabourin L, Lartigue C (2008) Surface topography in ball end milling process: Description of a 3D surface roughness parameter. Journal of Materials Processing Technology 195(1 - 3):135 - 143, DOI 10.1016/j.jmatprotec.2007.04.129

20. Quinsat Y, Lavernhe S, Lartigue C (2011) Characterization of 3D surface topography in 5-axis milling. Wear 271(3 - 4):590 - 595, DOI 10.1016/j.wear.2010.05.014, the 12th International Conference on Metrology and Properties of Engineering Surfaces

21. Souto-Lebel A, Guillemot N, Lartigue C, Billardon R (2011) Characterization and influence of defect size distribution induced by ball-end finishing milling on fatigue life. Procedia Engineering 19:343 - 348, DOI 10.1016/j.proeng.2011.11.123, 1st CIRP Conference on Surface Integrity (CSI)

22. Ungar PS, Brown CA, Bergstrom TS, Walker A (2003) Quantification of dental microwear by tan- 
dem scanning confocal microscopy and scale-sensitive fractal analyses. Scanning 25(4):185-193, DOI 10.1002/sca.4950250405

23. Zhang WH, Tan G, Wan M, Gao T, Bassir D (2008) A new algorithm for the numerical simulation of machined surface topography in multiaxis ball-end milling. Journal of Manufacturing Science and Engineering 130(1):011,003 1-11, DOI 10.1115/1.2815337 